\title{
Idiopathic pulmonary haemosiderosis revisited
}

\author{
O.C. Ioachimescu*, S. Sieber", A. Kotch
}

Idiopathic pulmonary haemosiderosis revisited. O.C. Ioachimescu, S. Sieber A. Kotch. C) ERS Journals Ltd 2004.

ABSTRACT: Idiopathic pulmonary haemosiderosis is a rare cause of diffuse alveolar haemorrhage of unknown aetiology. It occurs most frequently in children, has a variable natural history with repetitive episodes of diffuse alveolar haemorrhage, and has been reported to have a high mortality. Many patients develop iron deficiency anaemia secondary to deposition of haemosiderin iron in the alveoli.

Examination of sputum and bronchoalveolar lavage fluid can disclose haemosiderinladen alveolar macrophages (siderophages), and the lung biopsy shows numerous siderophages in the alveoli, without any evidence of pulmonary vasculitis, nonspecificl granulomatous inflammation, or deposition of immunoglobulins. Contrary to earlier reports, corticosteroids alone or in combination with other immunosuppressive agents may be effective for either exacerbations or maintenance therapy of idiopathic pulmonary haemosiderosis.

Eur Respir J 2004; 24: 162-170.
*Dept of Pulmonary Allergy and Critical Care Medicine, Cleveland Clinic foundation, Cleveland, $\mathrm{OH}$, and "Dept of Pathology and "Dept of Pulmonary Medicine and Critical Care, Danbury Hospital, Yale School of Medicine, Danbury, CT, USA.

Correspondence: O.C. Ioachimescu, 9500 Euclid Ave A90, Cleveland OH, 44195, USA.

Fax: 12164458160

E-mail: oioac@yahoo.com

Keywords: Azathioprine, diffuse alveolar haemorrhage, idiopathic pulmonary haemosiderosis, immunosuppressants, interstitial lung disease.

Received: December 132002

Accepted after revision: February 102004
Bleeding into the lower respiratory tract can be a severe, life-threatening condition. Diffuse alveolar haemorrhage (DAH) is characterised by haemoptysis, dyspnoea, alveolar infiltrates on chest radiograph, and various degrees of anaemia. Following a bleeding episode the alveolar macrophages convert the haemoglobin's iron into haemosiderin within 36-72 h [1, 2], hence the term haemosiderosis. Since the haemosiderin-laden macrophages reside for up to 4-8 weeks in the lungs [1-3], the term pulmonary haemosiderosis should be reserved for persistent or recurrent intra-alveolar bleeding. Conditions associated with DAH are very diverse (see Appendix). In some patients the work-up for a specific aetiology is negative and diagnosis of idiopathic pulmonary haemosiderosis (IPH) is rendered. The entity of IPH was initially described by VIRCHOW [4] in 1864 as "brown lung induration". A more in-depth characterisation of IPH was made in 1931, by CEELEN [5], who published the autopsy findings in two children who were found with large amounts of haemosiderin in the lungs. WALDENSTROM [6] established the first ante mortem diagnosis in 1944 [7]. The clinical triad of haemoptysis, pulmonary infiltrates and anaemia with no other identified cause (see Appendix) is considered diagnostic [8] if the lung biopsy excludes vasculitis/capillaritis, granulomas, or immune depositions [9].

\section{Case one}

Case one was a 37-yr-old male, nonsmoker, who was initially seen by a pulmonologist for acute respiratory insufficiency. He was born prematurely at 32 weeks of gestation, and had delayed neonatal developmental milestones. The patient was diagnosed with iron deficiency anaemia at aged 8 months. Gastrointestinal work-up excluded any source of bleeding. He was treated with iron supplements, and occasionally required blood transfusions. Subsequently, he underwent a bone marrow biopsy, which revealed findings consistent with iron-deficiency anaemia. When he was aged 2 yrs he developed cough, wheezing and dyspnoea, and a diagnosis of asthma was made. These symptoms recurred over the years with intermittent episodes of mild haemoptysis, until the patient was aged $12 \mathrm{yrs}$, when he was hospitalised for severe anaemia (haemoglobin $5.6 \mathrm{~g} \cdot \mathrm{dL}^{-1}$ ), severe haemoptysis and respiratory failure, necessitating intubation and mechanical ventilation. At that time, chest radiograph showed a background of interstitial reticular pattern with multiple alveolar-type opacities. The pulmonary function tests (PFTs) were consistent with a mild restrictive ventilatory defect and mild impairment of diffusing capacity. The electrocardiogram and the echocardiogram were both normal. Laboratory investigation revealed iron-deficiency anaemia, normal renal and liver function, negative work-up for antinuclear antibodies (ANA), rheumatoid factor (RF), antineutrophil cytoplasmic antibodies (ANCA), antiglomerular basement membrane antibodies (antiGBM), antigliadin, antireticulin and $\mathrm{cow}^{\prime} \mathrm{s}$ milk precipitins. He underwent an open lung biopsy, which showed the following features (fig. 1). 1) Dark-brownish macroscopic appearance of the lung biopsy tissue. 2) Alveoli, alveolar sacs, ducts, and bronchioles almost completely filled with haemosiderin-laden macrophages; hyperplasia of the alveolar epithelium. 3) Mild interstitial fibrosis, with disruption of the alveolar elastic fibres and massive encrustation with iron (fig. 2); no granulomatous reaction could be seen. 4) The pulmonary vessels showed mild thickening of their walls and minimal collection of lymphocytes in the perivascular interstitial connective tissue, without necrotising vasculitis.

A diagnosis of idiopathic pulmonary haemosiderosis (IPH) was made. The patient was started on prednisone $60 \mathrm{mg} \cdot \mathrm{day}^{-1}$ 


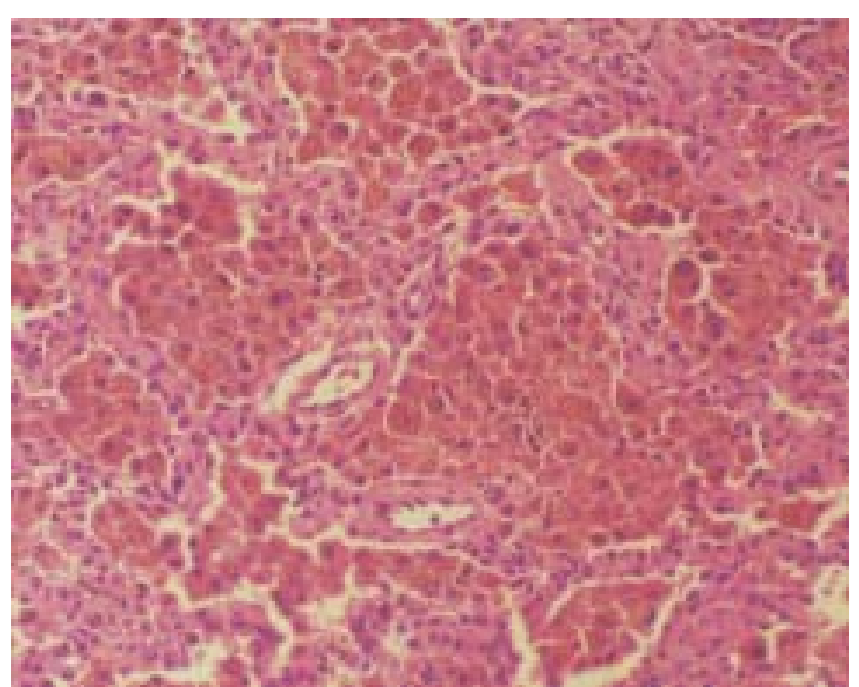

Fig. 1.-Open lung biopsy, showing mild nonspecific thickening of alveolar septae and numerous haemosiderin-laden macrophages within the alveolar spaces (haematoxylin and eosin stain).

and azathioprine $50 \mathrm{mg} \cdot \mathrm{day}^{-1}$, with prompt clinical response. During the next 24 yrs of follow-up he had a decreasing number of IPH exacerbations and required hospitalisation on three occasions for hypoxemic respiratory failure. Overall, he was treated without significant side-effects with azathioprine (continuously, 50-100 mg.day ${ }^{-1}$ ) for $11 \mathrm{yrs,} \mathrm{and} \mathrm{with} \mathrm{Pre-}$ dnisone (on-and-off, up to $60 \mathrm{mg} \cdot$ day $^{-1}$ ) for $18 \mathrm{yrs}$. He is now on a low-dose prednisone, and the last IPH exacerbation was 3 yrs ago, consisting of dyspnoea, cough, haemoptysis and hypoxemia. His level of activity is only minimally impaired, being overall very active. His last cardio-pulmonary exercise test showed a moderate decrease in the exercise tolerance, but with pulmonary vascular limitation and significant exertional desaturation.

\section{Case two}

Case two was a 37-yr-old male, nonsmoker, who was diagnosed with IPH by lung biopsy at aged 3 yrs. He had

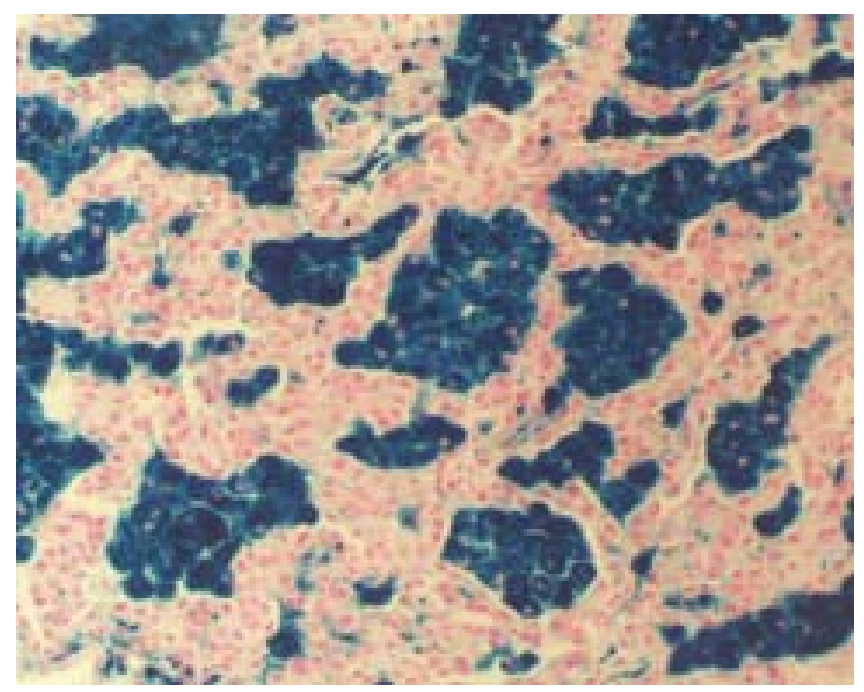

Fig. 2.-Iron stain performed on open-lung biopsy, highlighting the abundant intra-alveolar siderophages (prussian blue stain). multiple IPH exacerbations until he was aged $12 \mathrm{yrs}$, consisting of dyspnoea, cough and wheezing. He was treated with a few short courses of corticosteroids, with excellent clinical response. He was symptom-free for the next $6 \mathrm{yrs}$; his last IPH exacerbation was when he was aged 12 yrs. At the time of the diagnosis he presented with nonproductive cough and moderate exertional limitation. No fever, upper respiratory tract symptoms, haemoptysis, pleuritic chest pain, abdominal or urinary symptoms were reported. The chest radiograph showed new alveolar opacities in the right upper and left lower lobes together with (old) increased interstitial markings, consisting of reticular and fine micronodular calcified opacities scattered in both lung fields (fig. 3). The physical examination revealed fine inspiratory and expiratory crackles; no clubbing, no cyanosis nor signs of heart failure were identified. An induced sputum specimen showed multiple macrophages containing iron pigment, and no pathogenic bacteria or leukocytes were noted. The room-air arterial blood gas analysis on admission showed: $\mathrm{Pa}, \mathrm{O}_{2} 9.04 \mathrm{Kpa}$ $(68 \mathrm{mmHg})$, and $\mathrm{Pa}, \mathrm{CO}_{2} 5.32 \mathrm{Kpa}(40 \mathrm{mmHg})$; electrolytes, renal and liver functions were all normal. The haematocrit was $42 \%$, with a mean corpuscular volume of $85 \mathrm{fL}$, and the immunologic panel was normal (including erythrocyte sedimentation rate, complement, C-reactive protein, serum protein electrophoresis, antiGBM, ANCA, ANA, RF, antigliadin, antireticulin and cow-milk precipitins).

The diagnosis of IPH was made by open lung biopsy. The patient was started on a 4-week tapering-course of glucocorticoids, with good clinical and radiological response. The PFTs showed a mild restrictive ventilatory defect with a carbon dioxide diffusing capacity of the lung $(D \mathrm{~L}, \mathrm{CO})$ of $135 \%$ predicted, consistent with IPH and intercurrent DAH. He remained asymptomatic and without any further functional decline on follow-up PFTs for the next $19 \mathrm{yrs}$. He participated very successfully in several competitive sports, including football and wrestling. Currently, he is a married successful businessman with two children, active and without any functional limitation; he is not taking any medications.

\section{Epidemiology}

IPH is a rare condition with an unknown incidence and prevalence in the population. To date there are $\sim 500$ cases reported in the literature, either as isolated cases, or case series. Many patients previously reported as having IPH are probably misdiagnosed DAH cases, and with the advent of newer diagnostic tools and disease markers, they would currently be categorised differently (see Appendix). The rarity of the disease makes the prospective studies almost impossible. A Swedish study, published in 1984, and based on records reviewed between 1950-1979, estimated an incidence of 0.24 per million children per year [10], while a Japanese retrospective study [11], estimated $\sim 1.23$ cases per million yearly. In a biopsy study of interstitial lung diseases in children, the authors found lesions of IPH in $8 \%$ of cases [12].

Altogether, $80 \%$ of cases occur in children, most of them being diagnosed in the first decade of life $[8,9]$. The remaining $20 \%$ of cases are adult-onset IPH, but an unknown fraction is actually undiagnosed childhood-onset IPH; most of these cases are diagnosed before aged 30 yrs. Sex distribution appears to be balanced in childhood-onset IPH, and slightly skewed towards a male predominance in adult-onset IPH [9, 13]. Familial clustering has been described in several reports [14-18] suggesting a possible genetic or environmental contribution to the disease occurrence. 

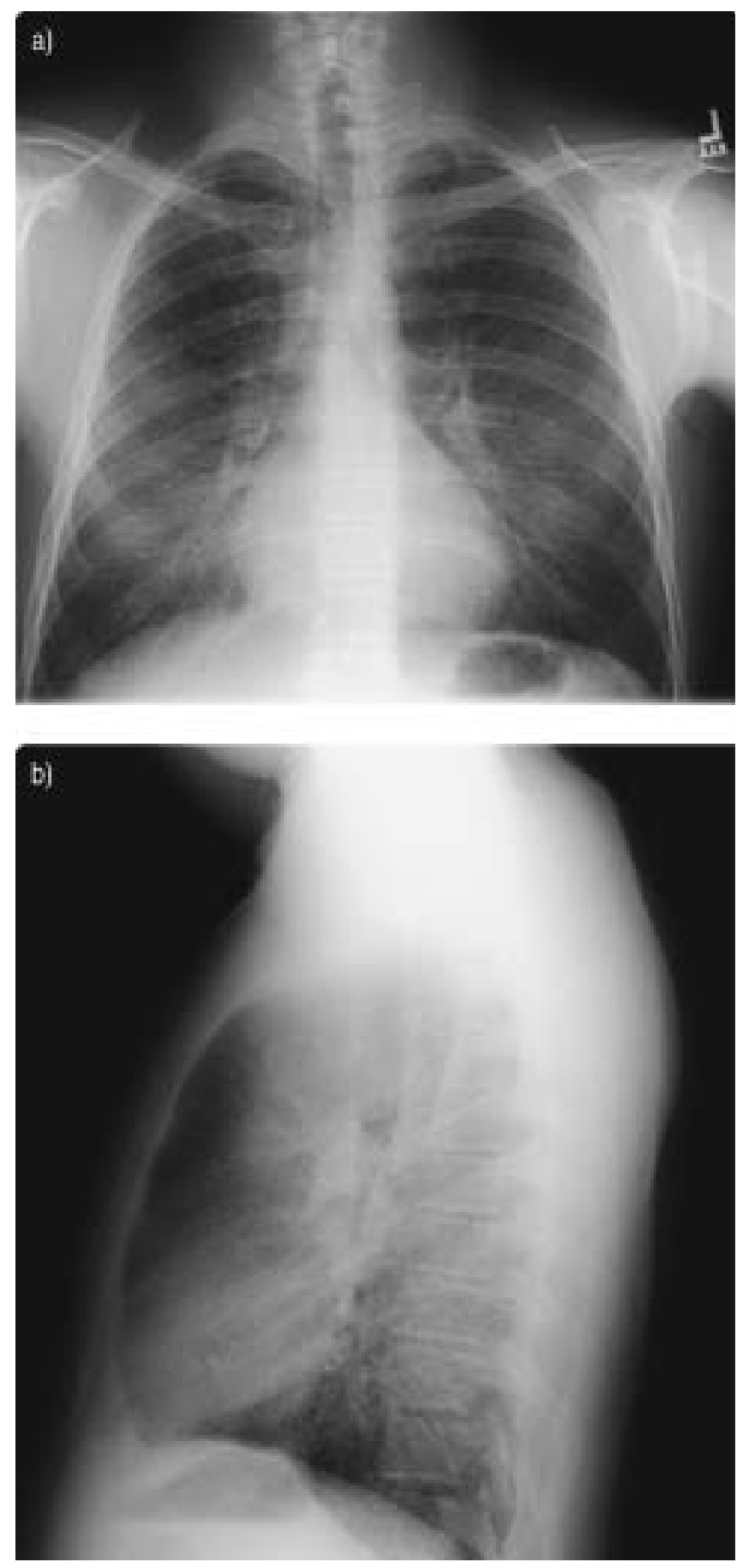

Fig. 3. -a) Postero-anterior and b) lateral baseline chest radiographs, illustrating reticular and fine micronodular opacities bilaterally.

\section{Aetiopathogenesis}

The aetiology of IPH remains unknown. There are a few aetiologic hypotheses as discussed below, the common delineator being postulated structural lesions of the alveolarendothelial membrane.

\section{Genetic theory}

Familial clustering of IPH has been described, suggesting hereditary inheritance versus a genetic predisposition to the influence of some unidentified environmental agents [14, 15,
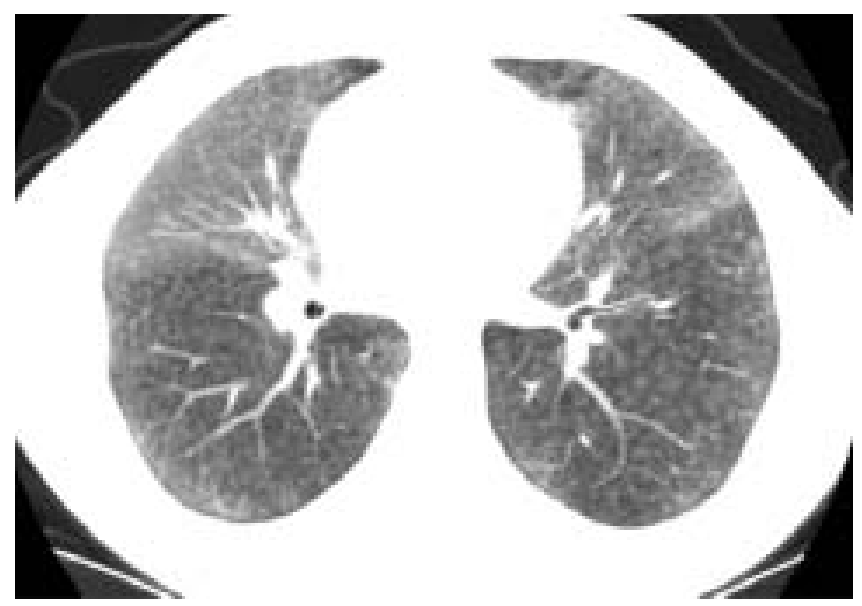

Fig. 4.-a) Computed tomography scan of the chest in idiopathic pulmonary haemosiderosis. Areas of ground-glass attenuation representing alveolar haemorrhage (more pronounced in lingula and middle lobe) and reticular micronodular appearance in the rest of the section, with moderate subpleural fibrosis.

19, 20]. The latest advances in genetics will soon create a new era in understanding the pathogenesis of the disease.

\section{Autoimmune theory}

Multiple ultrastructural alveolar membrane lesions were identified in patients with IPH in the initial era of electron microscopy: vacuolisation of the alveolar endothelial cells, focal thickening and scattered ruptures of the alveolar capillary basement membranes [21-26], though most of them antedate the appearance of highly sensitive immunological essays to better characterise other autoimmune conditions. Besides, these findings were not confirmed by all authors [27, 28]. Some believed in an autoimmune aetiopathogenesis based on the demonstration of plasma circulating immune complexes [29], though the immunohistochemical examination of lung tissue generally has not supported an immunological pathogenesis [23, 24, 27-29]. Interestingly, about a quarter of the patients who survive $>10 \mathrm{yrs}$ with this disease subsequently develop some form of autoimmune disease [30-32]. The accumulation of neutrophils in the alveoli also may play a role [33].

\section{Allergic theory}

A few children with IPH had detectable plasma antibodies (precipitins and immunoglobulin E (IgE)) against cow's milk, which led to the hypothesis of a systemic allergic reaction to milk components $[19,34-36]$; others failed to reproduce these findings [9, 29].

Other reports identified patients with both pulmonary haemosiderosis and coeliac disease [37-44]; to date there are $>10$ cases reported in the literature of coeliac disease and IPH and complete remission after instituting a gluten-free diet; this may be simply a coincidental association, highlighted by the rarity of both conditions, though a common pathogenic mechanism cannot be excluded.

\section{Environmental theory}

CAssimos et al. [16] and KAYSER et al. [45] have both proposed an association between the occurrence of IPH and 
exposure to insecticides, but this theory has never been proven. A series of articles linking environmental exposure to fungi (especially Stachybotrys atra) in water-damaged houses in Cleveland, $\mathrm{OH}$, USA and infantile pulmonary haemosiderosis led to an extensive investigation of possible infectious or mycotoxigenic pathogenesis [46-58]. The postulated mechanism is that fungal toxins called trichotecens, which are potent protein synthesis inhibitors, impede the angiogenesis underneath the rapidly forming alveolar membranes, making the acinar region prone to bleeding. In animal stachybotryomycotoxicosis, the alveolar haemorrhage is associated with significant inflammation, which can ultimately justify the empiric use of corticosteroids. Of interest is that glucocorticoids seemed to help in the management of the alveolar haemorrhage of these cases. Other moulds, like Alternaria, Aspergillus, Penicillium and Trichoderma spp., have also been invoked, but a clear relationship with IPH was not established.

Subsequent review by the Centre of Disease Control and Prevention (CDC; Cleveland) of the $S$. atra link questioned both the association with the exposure to moulds, i.e. S. atra (or S. chartarum) and the diagnosis of haemosiderosis, a better terminology being (recurrent or smoldering) intraalveolar haemorrhage [49]. The potential overlap of this disorder with other forms of pulmonary haemosiderosis has yet to be determined.

\section{Metabolic theory}

A defect in iron metabolism was postulated to be present in IPH [59]. Iron metabolism plays a crucial role in the biology of the respiratory system. The reticulo-endothelial system is the primary storage organ for iron, and its role in iron homeostasis is yet not entirely elucidated. Tissue macrophages are heterogeneous in their capacity to uptake extracellular ion, reprocess haemoglobin iron and degrade the senescent erythrocytes. In vitro macrophages derived from circulating monocytes phagocyte more than six times as many erythrocytes as pulmonary macrophages. Furthermore, the alveolar macrophages are less able to process and release $\mathrm{Fe}$ as the other types of macrophages [60]. The limited capacity of alveolar macrophages to metabolise haemoglobin can be related to absence of haeme oxygenase, an enzyme that can be induced following the phagocytosis of alveolar erythrocytes, as shown by MCGowAN et al. [61]. The exact relationship between nitric oxide and iron metabolism in this condition is currently unknown.

The total amount of iron in the alveolar macrophages can be quantified using cytochemical (Perls), colorimetric (ferrozine), or particle-induced X-ray emission techniques [62, 63]. The iron sources in the lungs are: circulatory, where it is bound to transferrin or lactoferrin, inhalatory (from cigarette smoke or metallic dusts); or from the red cell metabolism during episodes of alveolar haemorrhage. Transferrin is capable of providing iron only to cells that express CD71, or transferrin receptors, such as B and $\mathrm{T}$ lymphocytes and alveolar macrophages [62, 64, 65].

In DAH the alveolar macrophages accumulate large amounts of iron in short periods of time; acutely, the iron quickly saturates intra-cytoplasmic ferritin, and the extrairon, which will remain unbound to ferroproteins, can launch the sequence of cellular oxidative injury [66]. Later, the iron derived from the metabolism of haeme is found as trapped iron in haemosiderin (50-2000 times normal values) and, to a lesser extent, bound to other proteins (ferritin, etc.). Concomitantly with acute iron overload there is a significant increase in the alveolar macrophages synthesis of L (light)-type isoferritin, which, by an unknown mechanism, escapes into the systemic circulation, possibly by alveolar macrophage death or as a lymphocytic source [67]; furthermore, this amount of plasma ferritin does not seem to correlate with total-body iron deficiency status in IPH [68]. Also, the alveolar macrophage apparatus is easily exhaustible, and free iron can be present in the alveoli, leading eventually to pulmonary fibrosis $[62,69,70]$. One of the proposed toxic mechanism of the free iron ion against the pulmonary residing cells is thought to be related to its capacity to produce highly reactive hydroxyl radicals from less toxic oxygen superoxide and hydrogen peroxide, leading ultimately to lipid layer peroxidation, protein and carbohydrate degradation, and stimulation of fibrogenesis. The same mechanism was postulated to occur after lung transplantation [71]. It is unclear if the metabolic "arrest" within the alveolar macrophages occur at the level of haeme oxygenase involved in the degradation of haemoglobin in the endoplasmic reticulum, at the level of ferroportin 1, ceruloplasmin, hephaestin (or other proteins involved in the iron ion egress from the macrophages), or at a different site, and what is the therapeutic implication of the pathogenetic mechanism.

\section{Clinical presentation}

Clinical onset varies significantly from acute, fulminant haemoptysis, to chronic cough and dyspnoea, repetitive haemoptysis, fatigue, or only asymptomatic anaemia [9]. In adults the respiratory symptoms can be more pronounced [72], while in children failure to thrive and anaemia (and less often haemoptysis) can be the presenting findings.

The clinical course includes two phases. First, an acute phase "IPH exacerbation", corresponds to intra-alveolar bleeding episodes, manifested by cough, dyspnoea, haemoptysis and sometimes respiratory failure. Almost $100 \%$ of the adults experience haemoptysis during the disease course. Secondly, the chronic phase is characterised by a slow resolution of previous symptoms, with or without treatment. The alternation of the two phases is largely unpredictable.

The physical examination also differs in the two clinical phases. The acute phase has a wide range of symptoms, from signs of respiratory failure, cough and haemoptysis, to signs of worsening anaemia and, at the other end of the spectrum, an absolutely normal examination. The chronic phase includes pallor, emaciation, failure to thrive, hepato-splenomegaly and sometimes a normal examination. In those with fibrosis, bilateral crackles and clubbing may be present.

\section{Laboratory investigation}

The complete blood count will reveal variable degrees of anaemia, in the absence of quantitative or qualitative platelet defects, liver or kidney disease, coagulopathies, or any inflammatory syndromes. The sideropenic (transferrin saturation $<40 \%$ ), microcytic (MCV $<80 \mathrm{fL}$ ) anaemia is the consequence of DAH, with pulmonary sequestration of the iron bound to haemosiderrin. Plasma ferritin level can be normal or elevated because of the alveolar synthesis and release into the circulation and do not reflect the iron deposits of the body [68]. Bone marrow biopsy typically shows hyperplastic erythropoiesis, and low intramedullary iron stores [9, 19].

COHEN [33] defined three stages of IPH, based on clinical and laboratory findings: an acute phase, corresponding to the DAH episode, characterised by anaemia; a pre-acute phase (5-10 days prior to bleeding episode), with neutrophilia and eosinophilia; and a chronic phase. Regarding the pre-acute 
phase, it is questionable if this is not actually the subclinical phase of an intrapulmonic bleeding, with the well-known eosinophilic reaction.

Sputum examination, although not very sensitive, can demonstrate intra-alveolar bleeding (erythrocytes and haemosiderrin-laden macrophages), by haematoxylin-eosin and Prussian blue (Perl's) stains. If the patient is not spontaneously producing sputum, inducing it can be dangerous.

Bronchoalveolar lavage (BAL) from involved areas has a higher diagnostic yield than the sputum examination [73-76]. The predominant cellular types are the alveolar macrophages, filled with haemosiderrin, intact erythrocytes and occasionally neutrophils [33].

\section{Pulmonary function tests}

PFTs show in general a ventilatory restrictive pattern of variable severity $[19,77]$. The $D \mathrm{~L}, \mathrm{CO}$ can be low or normal during the chronic phase or elevated during the acute phase, as a manifestation of DAH [78]. Respiratory insufficiency can occur and can be either manifested at rest, or latent (only on exertion).

\section{Imaging studies}

First, there is no pathognomonic finding for IPH. There are a few radiological patterns closely related to the clinical phase. During the acute phase (IPH exacerbations) the chest radiographs show diffuse alveolar-type infiltrates, predominantly in the lower lung fields, with corresponding groundglass attenuation on the high-resolution computer axial tomography (CAT) scan (fig. 3 and 4) [79]. During the remission, the alveolar infiltrates tend to be reabsorbed and interstitial reticular and micronodular patterns of opacities ensue in the same areas, with variable degree of fibrosis [80]. ${ }^{99 \mathrm{~m}} \mathrm{Tc}$ - or ${ }^{51} \mathrm{Cr}$-based perfusion scans can demonstrate intraalveolar bleeding [81-86], but their utility in clinical practice remains extremely low.

\section{Diagnosis}

The diagnostic approach encompasses two stages. Stage one includes proof of diffuse intrapulmonary bleeding, based on the clinical picture, i.e. cough, dyspnoea, haemoptysis, pallor/failure to thrive, multiple alveolar-type opacities, secondary iron deficiency anaemia and numerous erythrocytes and siderophages in the sputum/BAL fluid from affected areas. Good-quality postero-anterior and lateral chest radiograms should be followed by thoracic high-resolution CT scans for better characterisation and location of the alveolar bleeding, and also evaluation of the degree of baseline interstitial fibrosis. A full evaluation of sideropenic anaemia includes gastrointestinal and genitourinary work-up for occult bleeding, iron studies showing low transferrin saturation $(<30 \%)$, low serum iron, microcytosis (MCV $<80 \mathrm{fL}$ ), with normal or high ferritin levels (as shown before, from the alveolar macrophages), low bone marrow deposits, and normal or high reticulocyte counts. PFTs include basic spirometry, plethismographic studies, and also DL,CO measurements.

The second stage of diagnosis is the exclusion of other diseases associated with DAH, i.e. autoimmune diseases, conditions associated with glomerulopathy, etc. (see below). This stage includes a lung biopsy, which does not show granulomas, capillaritis/vasculitis, or other organic lung disease. Besides the haematoxylin-eosin stains, specimens should be processed for immunofluorescence or immunohistochemistry to rule out any deposition of immunoglobulins and/or immune complexes. Transbronchial lung biopsy from the ground-glass attenuation areas on the CAT scan $(\leqslant 10$ samples) is the initial diagnostic procedure of choice. Larger samples can be obtained by video assisted thoracoscopic surgery or open lung biopsy. The laboratory investigation for ANA, antidouble-stranded DNA, ANCA (both perinuclear and cytoplasmic variants), antiGBM antibodies, antiphospholipid antibodies, IgG and IgE cow's milk antibodies, and RF should be negative. Also, patients can be tested for coeliac disease with plasma antigliadin and antireticulin antibodies, though the lack of gastro-intestinal symptoms makes this diagnosis unlikely.

\section{Pathology}

In IPH the lungs demonstrate the macroscopic finding of so-called "brown induration" [4], due to infiltration with iron and the various degrees of fibrosis. On light microscopy, the alveolar walls are thickened, the type two pneumocytes are hypertrophic and hyperplastic, and the interstitium contains collagen deposition in long-standing disease. Electron microscopy (not mandatory for diagnosis) can show swelling of the alveolar cells, with minimal thickenings and localised disruptions of the basement membranes, but no electron dense deposits in the alveolar basement membrane [21, 24, 27, 28, 87].

Most important for the diagnosis are three features visualised on light microscopy [9, 22, 24, 27, 28, 72]. First, the presence of intact or minimally fragmented erythrocytes in the distal airways and alveoli, as a reflection of recent/active DAH [9]. Secondly, multiple haemosiderin-laden macrophages (siderophages) as an expression of subacute/chronic or recurrent intra-pulmonary bleeding; they are best seen using Perls' reaction [9]. Staining for haemosiderrin (a complex of ferric iron, lipid, protein, and carbohydrate) is performed using Prussian blue reaction (Perl's reaction), which stains for ferric iron; the preparation reacts with potassium ferrocyanide to form a blue compound, ferriferrocyanide. Thirdly, the absence of any focal or diffuse smooth muscle cell proliferations, vascular malformations, malignancy, pulmonary infarcts, capillaritis/vasculitis, granulomatous inflammation, or infectious agents.

Useful ancillary tests are immunohistochemistry and immunofluorescence to exclude any intrapulmonary immunoglobulin or immune complex depositions. The value of electron microscopy remains for those situations when previous studies do not specifically rule out an immune deposition, particularly in the alveolar basement membrane.

\section{Treatment}

There are no controlled studies or good, large longitudinal surveys of patients with IPH, so the current state-of-the art of management of this disease is the result of observational studies on relatively small number of patients or incomplete data compiled over the years.

A number of therapeutical trials have been tried, including: 1) splenectomy, without significant results (there is no evidence of hypersplenism); and 2) systemic glucocorticoids, commonly started in the acute phase of IPH, with apparent good control and impact on mortality $[9,13,19,88]$, but with unclear effect on the chronic phase. The recommended starting dose is $\leqslant 1 \mathrm{mg} \cdot \mathrm{kg}^{-1} \cdot \mathrm{day}^{-1}$ of prednisolone for a couple of months, until the new alveolar infiltrates tend to resolve, and then a taper to off during the next months if 
symptoms do not recur. The majority of the patients with IPH seem to respond favorably to chronic oral corticosteroids, with a decreased number of IPH exacerbations and, possibly, a decline in fibrogenesis $[19,27,89,90]$. However, in children and adolescents, the long-term treatment can be problematic because of side-effects and a higher rate of recurrence on trial to taper/discontinue the steroids. Inhaled steroids also have been tried, but insufficient experience exists to date [91, 92]. Other immunosuppressant agents, including azathioprine, hydroxychloroquine, cyclophosphamide, and methotrexate, have been tried with variable results $[11,22,93-102]$. Among them, azathioprine in combination corticosteroids might be the best therapeutic regimen, especially in preventing IPH exacerbations. Only two cases of single-lung transplantation have been reported $[103,104]$. Both cases had clinical course complicated by IPH recurrence in the transplanted lungs. These two cases raise significant questions regarding this therapeutic option in IPH.

The clinical response to treatment can be quantified during the episodes of DAH as improvement in symptoms, anaemia, radiological infiltrates and the return to baseline of $D \mathrm{~L}, \mathrm{CO}$. Long-term follow-up should take into account the number and severity of haemorrhagic episodes and the progression of interstitial lung disease (the decline of $D \mathrm{~L}, \mathrm{CO}$ ).

\section{Prognosis}

The lack of prospective studies or large registries makes the evaluation of short and long-term prognosis difficult to assess. The most frequent cause of death is related to acute respiratory failure secondary to massive $\mathrm{DAH}$, or chronic respiratory failure and cor pulmonale due to severe pulmonary fibrosis $[9,33]$. In one case series of 68 patients with a mean follow-up of 4 yrs, 20 patients died, 17 had recurrent "IPH exacerbations", 12 had chronic active disease with persisting dyspnoea and anaemia, and 19 remained asymptomatic [9]. In that study, the average survival after the onset of symptoms was 2.5 yrs. Among the 68 patients diagnosed ante mortem, only $28(41 \%)$ were treated with steroids. Chryssanthopoulos et al. [105] studied the outcome of 30 children for an average of 5 yrs. The mean mortality rate was $60 \%$ and the mean survival was $\sim 3$ yrs (range, 3 months to $10.5 \mathrm{yrs}$ ). Almost $87 \%$ of the patients were on steroids at some point in time; none were treated with azathioprine [105]. In contrast, a different series of 17 paediatric patients, published in 1999 [90], showed a better prognosis, with a 5-yr survival rate of $86 \%$. The improved outcome might be related to a more prolonged immunosuppressive therapy in this study. It appears that children and adolescents have a more severe course and prognosis, while the adults have a more protracted course, with milder symptoms and a more favorable prognosis [19]. The cases presented here seem to show a drift in the outcome of these patients, either after prolonged immunosuppression, or spontaneous remission during a better care era.

\section{Conclusion}

Idiopathic pulmonary haemosiderosis is a rare disease of unknown aetiology, characterised by recurrent episodes of diffuse alveolar haemorrhage and sideropenic anaemia, which occurs most commonly in children. During an acute episode, a constellation of cough, dyspnoea, haemoptysis, with alveolar infiltrates and worsening anaemia, should raise suspicion for intrapulmonary bleeding. Sputum, bronchoalveolar lavage and eventually lung bioptic examination show numerous haemosiderin-laden macrophages in the alveoli, without evidence of capillaritis/vasculitis, granulomatous inflammation, deposition of immunoglobulins or immune complexes. Although it is unclear if idiopathic pulmonary haemosiderosis is an autoimmune disease, glucocorticoids and other immunosuppressive drugs seem to be effective during idiopathic pulmonary haemosiderosis exacerbations, and, possibly, also during the remission phase. The significant improvement in its morbidity and mortality during the past several decades is possibly due to the long-term use of immunosuppressant therapy.

\section{Appendix 1: Causes of diffuse alveolar haemorrhage}

Conditions associated with pulmonary vasculitis/capillaritis: Systemic vasculitides: microscopic polyangiitis, Wegener's granulomatosis, pauci immune glomerulonephritis, HenochSchonlein purpura, Behcet disease, systemic lupus erythematosus, rheumatoid arthritis, polymyositis, scleroderma, mixed connective tissue disorder, Churg Strauss syndrome, essential cryoglobulinemia, hypersensitivity vasculitis.

Other conditions: isolated pulmonary pauci-immune capillaritis, Goodpasture's syndrome, IgA nephropathy, antiphospholipid syndrome, autologous/allogeneic haematopoietic stem cell transplant, lung transplant rejection, ulcerative colitis, retinoic acid syndrome, subacute bacterial endocarditis.

Conditions not associated with pulmonary vasculitis/ capillaritis: coagulopathies, platelet functional defects, thrombocytopenia, diffuse intravascular coagulation, venous thromboembolic disease, pulmonary neoplasia, pulmonary veno-occlusive disease, pulmonary infarction, arterio-venous malformation, lymphangioleiomyomatosis, tuberous sclerosis, pulmonary capillary haemangiomatosis, mitral stenosis, diffuse alveolar damage, crack cocaine or trimellitic anhydride inhalation, idiopathic pulmonary haemosiderosis.

\section{References}

1. Sherman JM, Winnie G, Thomassen MJ, Abdul-Karim FW, Boat TF. Time course of hemosiderin production and clearance by human pulmonary macrophages. Chest 1984; 86: 409-411.

2. Epstein CE, Elidemir O, Colasurdo GN, Fan LL. Time course of hemosiderin production by alveolar macrophages in a murine model. Chest 2001; 120: 2013-2020.

3. Ghio AJ, Richards JH, Crissman KM, Carter JD. Iron disequilibrium in the rat lung after instilled blood. Chest 2000; 118: 814-823.

4. Virchow R. Die krankhaften Geshwulste. Berlin, August Hirschwald, 1864, pp. 26-240.

5. Ceelen W. Die kreislaufstorungen der Lungen [The circulatory Disorders of the Lungs]. In: Henke L, Lubarsch O, eds. Handbuch der speziellen pathologischen Anatomie und Histologie. Berlin, Springer-Verlag, 1931; pp. 20-163.

6. Waldenstrom J. Relapsing, diffuse, pulmonary bleeding or hemosiderosis pulmonum - a new clinical diagnosis. Acta Radiol 1944; 25: 33-42.

7. Heiner D. Pulmonary hemosiderosis. In: Chernick V, Kendig EJ, eds. Disorders of the respiratory tract in children. Philadelphia, PA, WB Sanders, 1990; pp. 498-509.

8. Morgan PG, Turner-Warwick M. Pulmonary haemosiderosis and pulmonary haemorrhage. Br J Dis Chest 1981; 75: 225-242.

9. Soergel K, Sommers SC. Idiopathic pulmonary hemosiderosis and related syndromes. Am J Med 1962; 32: 499-511.

10. Kjellman B, Elinder G, Garwicz S, Svan H. Idiopathic pulmonary haemosiderosis in Swedish children. Acta Paediatr Scand 1984; 73: 584-588. 
11. Ohga S, Takahashi K, Miyazaki S, Kato H, Ueda K. Idiopathic pulmonary haemosiderosis in Japan: 39 possible cases from a survey questionnaire. Eur J Pediatr 1995; 154: 994-995.

12. Boccon-Gibod L, Couvreur J. Results of lung biopsy in interstitial pneumopathies in children. A report on 100 cases. Ann Med Interne (Paris) 1979; 130: 501-506.

13. Bronson SM. Idiopathic pulmonary hemosiderosis in adults: report of a case and review of literature. Am J Roentgenol 1960; 83: 260-273.

14. Beckerman RC, Taussig LM, Pinnas JL. Familial idiopathic pulmonary hemosiderosis. Am J Dis Child 1979; 133: 609-611.

15. Thaell JF, Greipp PR, Stubbs SE, Siegal GP. Idiopathic pulmonary hemosiderosis: two cases in a family. Mayo Clin Proc 1978; 53: 113-118.

16. Cassimos CD, Chryssanthopoulos C, Panagiotidou C. Epidemiologic observations in idiopathic pulmonary haemosiderosis. J Pediatr 1983; 102: 698-702.

17. Choremis CB, Messaritakis JM, Karpouzas JG. Idiopathic pulmonary haemosiderosis. Lancet 1965; 10: 499.

18. Matsaniotis N, Karpouzas J, Apostolopoulou E, Messaritakis J. Idiopathic pulmonary haemosiderosis in children. Arch Dis Child 1968; 43: 307-309.

19. Ali A, Milman N, Clausen PP. Idiopathic pulmonary haemosiderosis. Favourable effect of corticosteroids in two women aged 16 and 55 years. Eur Respir Topic 1998; 4: 5357.

20. Breckenridge RL Jr, Ross JS. Idiopathic pulmonary hemosiderosis: a report of familial occurrence. Chest 1979; 75: 636-639.

21. Hyatt RW, Adelstein ER, Halazun JF, Lukens JN. Ultrastructure of the lung in idiopathic pulmonary hemosiderosis. Am J Med 1972; 52: 822-829.

22. Yeager H Jr, Powell D, Weinberg RM, Bauer H, Bellanti JA, Katz S. Idiopathic pulmonary hemosiderosis: ultrastructural studies and responses to azathioprine. Arch Intern Med 1976; 136: $1145-1149$

23. Dolan CJ Jr, Srodes CH, Duffy FD. Idiopathic pulmonary hemosiderosis. Electron microscopic, immunofluorescent, and iron kinetic studies. Chest 1975; 68: 577-580.

24. Corrin B, Jagusch M, Dewar A, et al. Fine structural changes in idiopathic pulmonary hemosiderosis. J Pathol 1987; 153: 249-256.

25. Gonzalez-Crussi F, Hull MT, Grosfeld JL. Idiopathic pulmonary hemosiderosis: evidence of capillary basement membrane abnormality. Am Rev Respir Dis 1976; 114: 689698.

26. Elliott ML, Kuhn C. Idiopathic pulmonary hemosiderosis. Ultrastructural abnormalities in the capillary walls. Am Rev Respir Dis 1970; 102: 895-904.

27. Irwin RS, Cottrell TS, Hsu KC, Griswold WR, Thomas HM III. Idiopathic pulmonary hemosiderosis: an electron microscopic and immunofluorescent study. Chest 1974; 65: $41-45$.

28. Donald KJ, Edwards RL, McEvoy JD. Alveolar capillary basement membrane lesions in Goodpasture's syndrome and idiopathic pulmonary haemosiderosis. Am J Med 1975; 59: 642-649.

29. Blanco A, Solis P, Gomez S, Linares P, Sanchez VE. Clqbinding immune complexes and other immunological studies in children with pulmonary hemosiderosis. Allergol Immunopathol (Madr) 1984; 12: 37-44.

30. Le Clainche L, Le Bourgeois M, Fauroux B, et al. Long-term outcome of idiopathic pulmonary hemosiderosis in children. Medicine (Baltimore) 2000; 79: 318-326.

31. Iijima $\mathrm{N}$, Torii $\mathrm{Y}$, Ito $\mathrm{S}$, et al. A case of idiopathic pulmonary hemosiderosis recurrent after remission of fifteen years and associated with Sjogren's syndrome. Nihon Kyobu Shikkan Gakkai Zasshi 1988; 26: 1191-1194.

32. Lemley DE, Katz P. Rheumatoid-like arthritis presenting as idiopathic pulmonary hemosiderosis: a report and review of the literature. J Rheumatol 1986; 13: 954-957.
33. Cohen S. Idiopathic pulmonary hemosiderosis. Am J Med Sci 1999; 317: 67-74.

34. Heiner D, Sears J, Kniker W. Multiple precipitins to cow milk in chronic respiratory disease. Am J Dis Child 1962; 103: 634-654.

35. Boat TF, Polmar SH, Whitman V, Kleinerman JI, Stern RC, Doershuk CF. Hyperreactivity to cow milk in young children with pulmonary hemosiderosis and cor pulmonale secondary to nasopharyngeal obstruction. J Pediatr $1975 ; 87$ : 23-29.

36. Torres MJ, Giron MD, Corzo JL, et al. Release of inflammatory mediators after cow's milk intake in a newborn with idiopathic pulmonary hemosiderosis. J Allergy Clin Immunol 1996; 98: 1120-1123.

37. Wright PH, Menzies IS, Pounder RE, Keeling PW. Adult idiopathic pulmonary haemosiderosis and coeliac disease. $Q$ $J$ Med 1981; 50: 95-102.

38. Hemoptysis, pulmonary infiltrates, and diarrhea in a 36year-old man. Am J Med 1986; 80: 930-938.

39. Reading R, Watson JG, Platt JW, Bird AG. Pulmonary haemosiderosis and gluten. Arch Dis Child 1987; 62: 513-515.

40. Mah MW, Priel IE, Humen DP, Brown NE, Sproule BJ. Idiopathic pulmonary hemosiderosis, complete heart block and celiac disease. Can J Cardiol 1989; 5: 191-194.

41. Pacheco A, Casanova C, Fogue L, Sueiro A. Long-term clinical follow-up of adult idiopathic pulmonary haemosiderosis and coeliac disease. Chest 1991; 99: 1525-1526.

42. Perelman S, Dupuy C, Bourrillon A. The association of pulmonary hemosiderosis and celiac disease. Apropos of a new case in a child. Ann Pediatr (Paris) 1992; 39: 185-188.

43. Ploier R, Emhofer J, Dorninger L, et al. Immunological aspects of a child with idiopathic pulmonary hemosiderosis and celiac disease. Klin Padiatr 1998; 210: 409-412.

44. Rieu D, Ariole P, Lesbros D, Emberger JM, Jean R. Idiopathic pulmonary hemosiderosis and celiac disease in a child. Case report. Presse Med 1983; 12: 2931-2933.

45. Kayser K, Plodziszewska M, Waitr E, Slodkowska J, Altiner M, Gabius HJ. Diffuse pulmonary hemosiderosis after exposure to pesticides. A case report. Respiration 1998; 65: 214-218.

46. Update: pulmonary hemorrhage/hemosiderosis among infants, Cleveland, Ohio, 1993-1996. MMWR 2000; 49: $180-184$

47. Acute pulmonary hemorrhage/haemosiderosis among infants, Cleveland, January 1993-November 1994. MMWR 1994; 43: 881-883.

48. Update: pulmonary hemorage/hemosiderosis among infants, Cleveland, Ohio, 1993-1996. MMWR 1997; 46: 33-35.

49. From the Centres for Disease Control and Prevention. Update: pulmonary hemorrhage/hemosiderosis among infants, Cleveland, Ohio, 1993-1996. JAMA 2000; 283: 1951-1953.

50. Montana E, Etzel RA, Allan T, Horgan TE, Dearborn DG Environmental risk factors associated with pediatric idiopathic pulmonary hemorrhage and hemosiderosis in a Cleveland community. Pediatrics 1997; 99: E5.

51. Etzel RA, Montana E, Sorenson WG, et al. Acute pulmonary hemorrhage in infants associated with exposure to Stachybotrys atra and other fungi. Arch Pediatr Adolesc Med 1998; 152: 757-762.

52. Dearborn DG, Smith PG, Dahms BB, et al. Clinical profile of 30 infants with acute pulmonary hemorrhage in Cleveland. Pediatrics 2002; 110: 627-637.

53. Dearborn DG, Yike I, Sorenson WG, Miller MJ, Etzel RA. Overview of investigations into pulmonary hemorrhage among infants in Cleveland, Ohio. Environ Health Perspect 1999; 107: Suppl 3, 495-499.

54. Jarvis BB, Sorenson WG, Hintikka EL, et al. Study of toxin production by isolates of Stachybotrys chartarum and Memnoniella echinata isolated during a study of pulmonary haemosiderosis in infants. Appl Environ Microbiol 1998; 64: $3620-3625$. 
55. Hodgson MJ, Morey $\mathrm{P}$, Leung WY, et al. Buildingassociated pulmonary disease from exposure to Stachybotrys chartarum and Aspergillus versicolor. J Occup Environ Med 1998; 40: 241-249.

56. Vesper SJ, Dearborn DG, Yike I, Sorenson WG, Haugland RA. Hemolysis, toxicity, and randomly amplified polymorphic DNA analysis of Stachybotrys chartarum strains. Appl Environ Microbiol 1999; 65: 3175-3181.

57. Vesper SJ, Vesper MJ. Stachylysin may be a cause of hemorrhaging in humans exposed to Stachybotrys chartarum. Infect Immun 2002; 70: 2065-2069.

58. Vesper SJ, Dearborn DG, Yike I, et al. Evaluation of Strachybotrys chartarum in the house of an infant with pulmonary hemorrhage: quantitative assessment before, during and after remediation. $J$ Urban Health 2000; 77: 68-85.

59. Fillet G, Beguin Y, Baldelli L. Model of reticuloendothelial iron metabolism in humans: abnormal behavior in idiopathic hemochromatosis and in inflammation. Blood 1989; 74: 844 851.

60. Custer G, Balcerzak S, Rinehart J. Human macrophage hemoglobin-iron metabolism in vitro. Am J Hematol 1982; 13: 23-36.

61. McGowan SE, Stone PJ, Snider GL, Franzblau C. Alveolar macrophage modulation of proteolysis by neutrophil elastase in extracellular matrix. Am Rev Respir Dis 1984; 130: 734-739.

62. Mateos F, Brock JH, Perez-Arellano JL. Iron metabolism in the lower respiratory tract. Thorax 1998; 53: 594-600.

63. Ghadially FN. Invited review. The technique and scope of electron-probe X-ray analysis in pathology. Pathology 1979; 11: 95-110.

64. Hirata T, Bitterman PB, Mornex JF, Crystal RG. Expression of the transferrin receptor gene during the process of mononuclear phagocyte maturation. J Immunol 1986; 136: 1339-1345.

65. Wyllie JC. A study of ferritin iron formation in the rabbit alveolar macrophage. Exp Cell Res 1973; 78: 98-102.

66. Wesselius LJ, Flowers CH, Skikne BS. Alveolar macrophage content of isoferritins and transferrin. Comparison of nonsmokers and smokers with and without chronic airflow obstruction. Am Rev Respir Dis 1992; 145: 311-316.

67. McGowan S, Power J. Effect of ice on bruising at cardiac catheter insertion sites (brachial approach). Aust J Adv Nurs 1988; 5: 27-32.

68. Milman N, Pedersen FM. Idiopathic pulmonary haemosiderosis. Epidemiology, pathogenic aspects and diagnosis. Respir Med 1998; 98: 902-907.

69. Castranova V, Bowman L, Miles PR, Reasor MJ. Toxicity of metal ions to alveolar macrophages. Am J Ind Med 1980; 1: 349-357.

70. Egan JJ, Martin N, Hasleton PS, et al. Pulmonary interstitial fibrosis and haemosiderin-laden macrophages: late following heart transplantation. Respir Med 1996; 90: 547-551.

71. Baz MA, Ghio AJ, Roggli VL, Tapson VF, Piantadosi CA. Iron accumulation in lung allografts after transplantation. Chest 1997; 112: 435-439.

72. Ioachimescu O. Idiopathic pulmonary hemosiderosis in adults. Pneumologia 2003; 52: 38-43.

73. Danel C, Israel-Biet D, Costabel U, Rossi GA, Wallaert B. The clinical role of BAL in pulmonary haemeorrhages. Eur Respir J 1990; 3: 951-959.

74. Danel C, Le Bourgeois M, De Blic J, Scheinmann P, Nezelof C. Anatomoclinical approach in idiopathic pulmonary haemosiderosis. Apropos of 12 cases. Arch Anat Cytol Pathol 1989; 37: 160-165.

75. Grebski E, Hess T, Hold G, Speich R, Russi E. Diagnostic value of hemosiderin-containing macrophages in bronchoalveolar lavage. Chest 1992; 102: 1794-1799.

76. Perez-Arellano JL, Losa Garcia JE, Garcia Macias MC, Gomez GF, Jimenez LA, de Castro S. Hemosiderin-laden macrophages in bronchoalveolar lavage fluid. Acta Cytol 1992; 36: 26-30.

77. Allue $\mathrm{X}$, Wise MB, Beaudry PH. Pulmonary function studies in idiopathic pulmonary hemosiderosis in children. Am Rev Respir Dis 1973; 107: 410-415.

78. Ewan PW, Jones HA, Rhodes CG, Hughes JM. Detection of intrapulmonary hemorrhage with carbon monoxide uptake. Application in goodpasture's syndrome. N Engl J Med 1976; 295: 1391-1396.

79. Akyar S, Ozbek SS. Computed tomography findings in idiopathic pulmonary hemosiderosis. Respiration 1993; 60: 63-64.

80. Buschman DL, Ballard R. Progressive massive fibrosis associated with idiopathic pulmonary hemosiderosis. Chest 1993; 104: 293-295.

81. DeGowin RL, Sorensen LB, Charleston DB, Gottschalk A, Greenwald JH. Retention of radioiron in the lungs of a woman with idiopathic pulmonary hemosiderosis. Ann Intern Med 1968; 69: 1213-1220.

82. Dutau G, Ghisolfi J, Rochiccioli P, Boneu A, Corberand J, Dalous A. Idiopathic pulmonary hemosiderosis. Apropos of a series of 7 cases. Diagnostic value of isotope exploration. Pediatrie 1972; 27: 647-667.

83. Gilly R, Farouz S, Mamelle JC, Dutruge J, Martal C. Idiopathic pulmonary hemosiderosis in a child (benefits of iron 59 isotopic exploration and electronic microscopy). Lyon Med 1972; 227: 1095-1104.

84. Kurzweil PR, Miller DR, Freeman JE, Reiman RE, Mayer $\mathrm{K}$. Use of sodium chromate $\mathrm{Cr} 51$ in diagnosing childhood idiopathic pulmonary hemosiderosis. Am J Dis Child 1984; 138: 746-748.

85. Miller T, Tanaka T. Nuclear scan of pulmonary hemorrhage in idiopathic pulmonary haemosiderosis. AJR 1979; 132: $120-121$.

86. Samuels LD, Bass JC. $51 \mathrm{Cr}$ lung scan in idiopathic pulmonary hemosiderosis. $J$ Nucl Med 1969; 10: 106-107.

87. Brambilla CG, Brambilla EM, Stoebner P, Dechelette E. Idiopathic pulmonary hemorrhage. Ultrastructural and mineralogic study. Chest 1982; 81: 120-123.

88. Walters GO, Miller FM, Worwood M. Serum ferritin concentration and iron stores in normal subjects. $J$ Clin Pathol 1973; 26: 770-772.

89. Kiper N, Gocmen A, Ozcelik U, Dilber E, Anadol D. Longterm clinical course of patients with idiopathic pulmonary hemosiderosis (1979-1994): prolonged survival with lowdose corticosteroid therapy. Pediatr Pulmonol 1999; 27: 180184.

90. Saeed MM, Woo MS, MacLaughlin EF, Margetis MF, Keens TG. Prognosis in pediatric idiopathic pulmonary haemosiderosis. Chest 1999; 116: 721-725.

91. Elinder G. Budesonide inhalation to treat idiopathic pulmonary haemosiderosis. Lancet 1985; 1: 981-982.

92. Tutor JD, Eid NS. Treatment of idiopathic pulmonary haemosiderosis with inhaled flunisolide. South Med J 1995; 88: 984-986.

93. Byrd RB, Gracey DR. Immunosuppressive treatment of idiopathic pulmonary hemosiderosis. JAMA 1973; 226: 458459.

94. Rossi GA, Balzano E, Battistini E, et al. Long-term prednisone and azathioprine treatment of a patient with idiopathic pulmonary hemosiderosis. Pediatr Pulmonol 1992; 13: $176-180$

95. Airaghi L, Ciceri L, Giannini S, Ferrero S, Meroni PL, Tedeschi A. Idiopathic pulmonary haemosiderosis in an adult. Favourable response to azathioprine. Monaldi Arch Chest Dis 2001; 56: 211-213.

96. Bagnato L, Grilli C, Portioli P, Biella C, Carnelli V. Longterm evaluation of immunosuppressive therapy in childhood idiopathic pulmonary haemosiderosis. Pediatr Med Chir 1986; 8: 671-674.

97. Berger G, Rupprecht E. Contribution to the treatment of idiopathic pulmonary hemosiderosis by means of immunosuppressive agents. Padiatr Grenzgeb 1978; 17: 213-220.

98. Carnelli V, Biraghi V, Zurlo MG, Rossi MR, Parziani V. Efficacy of treatment with cyclophosphamide in a case of 
idiopathic pulmonary hemosiderosis. Minerva Pediatr 1979; 31: 3893-3900.

99. Ceruti DE, Casar CC, Diaz CA, Jimenez dlJ, Vildosola SM, Torres-Goitia CJ. Idiopathic pulmonary hemosiderosis; long-term follow-up in 10 patients treated with corticoids and immunosuppressive agents. Bol Med Hosp Infant Mex 1980; 37: 985-1002.

100. Garatti M, Parravicini C, Meroni PL, Giulotto P, Rusconi $\mathrm{R}$, Carnelli V. Idiopathic pulmonary hemosiderosis: report of a case with a favorable response to cyclophophamide therapy. Pediatr Med Chir 1983; 5: 95-98.

101. Meral A, Gunay U, Kucukerdogan A, Canitez Y, Ozuysal S. Chloroquine in idiopathic pulmonary haemosiderosis. A case report. Turk J Pediatr 1997; 39: 111-115.
102. O'Donohue WJ, Jr. Idiopathic pulmonary haemosiderosis with manifestations of multiple connective tissue and immune disorders. Treatment with cyclophosphamide. Am Rev Respir Dis 1974; 109: 473-479.

103. Wroblewski BM, Stefanovic CR, McDonough VM, Kidik PJ. The challenges of idiopathic pulmonary hemosiderosis and lung transplantation. Crit Care Nurse 1997; 17: 39-44.

104. Calabrese F, Giacometti C, Rea F, et al. Recurrence of idiopathic hemosiderosis in a young adult patient after bilateral single-lung transplantation. Transplantation 2002; 74: 1643-1645.

105. Chryssanthopoulos C, Cassimos C, Panagiotidou C. Prognostic criteria in idiopathic pulmonary hemosiderosis in children. Eur J Pediatr 1983; 140: 123-125. 